\title{
Telessaúde como ferramenta na formação médica durante a pandemia da COVID-19: relato de experiência
}

\author{
Telehealth as a medical education tool during the COVID-19 pandemic: experience report
}

\author{
Matheus Augusto Obici Massucato' (D) matheus.massucato@ufms.br \\ Ana Luísa Green Ribeiro' (1) algr952@gmail.com

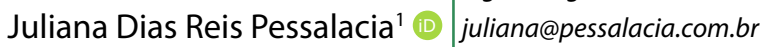 \\ Vinicius de Jesus Rodrigues Neves ${ }^{1}$ (D) viniciusjr.mfc@gmail.com \\ Vânia Paula Stolte-Rodrigues² (D) vania.stolte@gmail.com
}

\begin{abstract}
RESUMO
Introdução: A pandemia da Covid-19 interrompeu e desafiou a estrutura tradicional da educação médica, fundamentada no ensino presencial, e, como medida de apoio aos esforços dos órgãos governamentais para a redução dos riscos de disseminação da doença, tornaram-se necessários o distanciamento do atendimento médico/paciente e o aumento da oferta de serviços de telessaúde pelos sistemas de saúde. No Brasil, o modelo de telessaúde busca melhorar a qualidade do atendimento da atenção primária à saúde (APS), integrando ensino e serviço por meio de atividades de teleducação e teleassistência, tais como a teleconsultoria, a Segunda Opinião Formativa (SOF), a teleducação e o telediagnóstico. Assim, este artigo relata a experiência de estudantes de Medicina em ações de telessaúde durante a pandemia da Covid-19 no Brasil, buscando esclarecer as contribuições e limitações dessa experiência no processo ensino-aprendizado no contexto da formação médica.
\end{abstract}

Relato de experiência: A participação no projeto permitiu a vivência de diversas atividades de telessaúde sob a supervisão e orientação de docentes da área de saúde, além da produção de materiais informativos e educativos. As atividades propostas permitiram o aprimoramento do raciocínio clínico por meio da medicina baseada em evidências (MBE), principalmente no auxílio a teleconsultorias e perguntas frequentes.

Discussão: O uso de tecnologias tornou-se indispensável durante a pandemia, e, dentro desse cenário, um projeto de telessaúde mostrou-se como uma estratégia importante e eficaz para educação permanente entre profissionais e educação em saúde para a comunidade, evitando aglomerações e prevenindo a disseminação do vírus. Além disso, as ações de forma remota, como teleconsultorias, resoluções de perguntas frequentes e teleducação, mostraram ser uma estratégia importante de acesso à saúde não somente em tempos de pandemia.

Conclusão: Nossa experiência possibilitou fomentar o senso crítico e disseminar conteúdo de forma segura, técnica e baseada em evidências. O exercício do raciocínio clínico nos levou a uma experiência de grande valia e a crer que a inclusão da prática de telessaúde pode trazer ganhos importantes à grade curricular dos cursos de Medicina.

Palavras-chave: Telemedicina; Estudantes; Educação de Graduação em Medicina; Faculdades de Medicina; Infecções por Coronavírus.

\begin{abstract}
Introduction: The Covid-19 pandemic interrupted and challenged the traditional structure of medical education, based on face-to-face teaching, and, as a measure of support for the efforts of government agencies to reduce the risk of spreading the disease, distance became necessary. medical/ patient care and the increase in the supply of telehealth services by health systems. In Brazil, the telehealth model seeks to improve the quality of care in primary health care (PHC), integrating education and service through tele-education and tele-assistance activities, such as teleconsulting, the Second Formative Opinion (SOF), tele-education and telediagnosis. Thus, this article reports the experience of medical students in telehealth actions during the Covid-19 pandemic in Brazil, seeking to clarify the contributions and limitations of this experience in the teaching-learning process in the context of medical education.
\end{abstract}

Experience report: Participation in the project allowed the experience of various telehealth activities under the supervision and guidance of professors in the health area, in addition to the production of informative and educational materials. The proposed activities allowed for the improvement of clinical reasoning through evidence-based medicine (EBM), especially in helping teleconsultations and frequently asked questions.

Discussion: The use of technologies became indispensable during the pandemic, and, within this scenario, a telehealth project proved to be an important and effective strategy for continuing education among professionals and health education for the community, avoiding crowding and preventing the spread of the virus. In addition, remote actions, such as teleconsultations, resolution of frequently asked questions and tele-education, proved to be an important strategy for accessing health care not only in times of pandemic.

Conclusion: Our experience made it possible to foster critical thinking and disseminate content in a safe, technical and evidence-based way. The exercise of clinical reasoning led us to an experience of great value and to believe that the inclusion of the practice of telehealth can bring important gains to the curriculum of Medicine courses.

Keywords: Telemedicine; Students; Medical Education; Medical School; Coronavirus Infections.

1 Universidade Federal de Mato Grosso do Sul, Três Lagoas, Mato Grosso do Sul, Brasil.

2 Secretaria de Estado de Saúde de Mato Grosso do Sul, Três Lagoas, Mato Grosso do Sul, Brasil.

Editora-chefe: Rosiane Viana Zuza Diniz. Editor associado: Jorge Carvalho Guedes.

Recebido em 04/05/21; Aceito em 05/07/21. | | Avaliado pelo processo de double blind review. 


\section{INTRODUÇÃO}

A Organização Mundial da Saúde (OMS) e a American Telemedicine Association definem telemedicina como o uso de comunicações eletrônicas e tecnologias de informação na prestação de serviços clínicos de forma remota'. A telessaúde como projeto contempla um escopo mais amplo de serviços remotos de assistência, como treinamentos de fornecedores, reuniões administrativas e educação permanente, além de contemplar os serviços clínicos de telemedicina².

$\mathrm{O}$ atual contexto da pandemia da coronavirus disease 2019 (Covid-19) tem gerado estresse sem precedentes em gestores e profissionais dos sistemas de saúde e educação, criando desafios práticos e logísticos para o ambiente de aprendizagem que podem ter um impacto duradouro na educação médica ${ }^{3-5}$.

A nova pandemia interrompeu e desafiou a estrutura tradicional e bem estabelecida da educação médica de graduação e pós-graduação - cuja base de formação é o ensino presencial ${ }^{4}$. Como medida de apoio aos esforços dos órgãos governamentais para a redução dos riscos de disseminação da doença, tornaramse necessários o distanciamento do atendimento médico/ paciente durante a pandemia e o aumento da oferta de serviços de telessaúde pelos sistemas de saúde ${ }^{3}$.

O Conselho Federal de Medicina (CFM) por meio do Ofício no 1.756/2020 encaminhado ao Ministério da Saúde e da Portaria no 476, de 20 de março de 2020, buscou o aperfeiçoamento e a eficiência dos serviços médicos prestados em caráter excepcional e enquanto durarem ações de combate à Covid-19 no Brasil, por meio da prática ética da utilização da telemedicina para teleorientação, telemonitoramento e teleinterconsulta, com base na necessidade de proteger tanto a saúde dos médicos como a dos pacientes ${ }^{6}$.

Contudo, em outros países, como Austrália ${ }^{7}$ e França ${ }^{8}$, os serviços de telessaúde já se encontram regulamentados e com investimentos em tecnologias e incentivos governamentais. Nesses países, também têm sido incentivadas ações voltadas para a formação e o treinamento de profissionais e para a integração da temática nos currículos médicos.

No Brasil, o modelo de telessaúde busca melhorar a qualidade do atendimento da atenção primária à saúde (APS), de modo a integrar ensino e serviço por meio de atividades de teleducação e teleassistência, visando principalmente ao fortalecimento da Estratégia Saúde da Família (ESF)9. A telessaúde envolve diferentes abordagens, tais como a teleconsultoria, a Segunda Opinião Formativa (SOF), a teleducação e o telediagnóstico ${ }^{10}$.

Nesse cenário de avanço das ações de telessaúde, torna-se necessário o treinamento de alunos em ferramentas digitais, e a educação sobre suas limitações deve fazer parte do ensino de graduação. Devem-se também revisar os currículos de graduação em Medicina a fim preparar os futuros médicos para uma era dominante da telemedicina ${ }^{11}$. No contexto norte-americano, por exemplo, os estudantes de Medicina estão envolvidos em ações de telessaúde como parte de suas rotações clínicas, embora a experiência do estudante-consultor e os benefícios gerais para a sua formação ainda estejam escassamente descritos na literatura ${ }^{12}$. Assim, à medida que as escolas médicas se adaptam à nova cara da medicina, a transição dos alunos para a telemedicina deve ser priorizada, visto que ela terá um importante papel no futuro ${ }^{11}$.

A prática de telessaúde ainda é pouco contemplada nos currículos de formação médica no Brasil, e há uma literatura limitada sobre a experiência de estudantes de Medicina nessa modalidade de assistência. Por conta disso, este artigo relata a experiência de discentes de Medicina em ações de telessaúde durante a pandemia da Covid-19 no Brasil, com o propósito de esclarecer as contribuições e limitações dessa vivência no processo ensino-aprendizado no contexto da formação médica.

\section{RELATO DE EXPERIÊNCIA}

A aproximação dos alunos de Medicina às ações de telessaúde teve início a partir da participação em projeto de extensão aprovado em um edital extraordinário da Universidade Federal de Mato Grosso do Sul (UFMS) (Covid-19 - Projetos e Ideias - Pró-Reitoria de Pesquisa e Pó-Graduação (Propp), PróReitoria de Extensão, Cultura e Esporte (Proece) e Agência de Desenvolvimento, Inovação e Relações Internacionais (Aginova) da UFMS n²2/2020) voltado à proposição de projetos, ideias e ações para o enfrentamento dos desafios vividos pela sociedade em função da pandemia.

Assim, a proposta de um projeto de telessaúde no câmpus de Três Lagoas (CPTL/UFMS) surgiu a partir da percepção de docentes do curso de Medicina sobre a necessidade de se trabalhar o atual contexto mundial de avanços no domínio das tecnologias de informação e comunicação (TIC), as quais têm proporcionado o alcance de ações de teleducação e telemedicina por meio de redes sociais, aplicativos baseados na $w e b$, teleconsultas, teleconferências e abordagens multimídia (imagens digitais e de vídeo).

Entre maio e dezembro de 2020 (período afetado pela pandemia), a unidade desenvolveu ações de teleconsultoria assíncrona, teleducação e SOF, sendo as atividades desenvolvidas a partir da participação de dez estudantes de Medicina (de diferentes períodos) e de 18 docentes dos cursos de Medicina e Enfermagem.

No projeto de extensão da unidade de telessaúde do CPTL, propuseram-se os seguintes objetivos: a construção de vídeos e materiais educativos digitais sobre Covid-19 e a 
disponibilização deles a toda a população do estado, por meio de redes sociais, site das instituições parceiras e WhatsApp; a capacitação dos docentes e discentes da equipe para a realização de teleconsultorias e teleducação; a resolução de teleconsultorias enviadas por profissionais de saúde da APS de todo o estado; e a publicação de perguntas frequentes sobre Covid-19 na página do Núcleo de Telessaúde do estado de Mato Grosso do Sul. Atuamos sob a coordenação-geral de uma docente que era a responsável pelo planejamento das ações da unidade (CPTL) e pela mediação com os responsáveis no Núcleo Estadual de Telessaúde.

Assim, durante o período inicial de atividades da unidade, produziram-se:

- Doze vídeos curtos que foram publicados no Instagram do projeto (https://www.instagram.com/ tlsaudeufms/), na página do Núcleo de Telessaúde do estado no Facebook (https://www.facebook. com/tlsaudeufms/) e no site do Telessaúde MS (http://www .telessaude.saude.ms.gov.br/).

- Sessenta e oito teleconsultorias realizadas por profissionais de saúde da APS do estado de Mato Grosso do Sul.

- Dezessete perguntas da semana, as quais se encontram publicadas no site do Núcleo de Telessaúde do estado, acessível a todos os profissionais de saúde e à população em geral.

- Duas SOFs nas áreas de farmacologia e ginecologia e obstetrícia, as quais se encontram publicadas na página da Biblioteca Virtual em Saúde (BVS) e do Núcleo de Telessaúde do estado.

A tarefa de atender aos anseios e às dúvidas da comunidade iniciou-se com a proposta da montagem e divulgação de vídeos curtos voltados principalmente ao contexto da pandemia da Covid-19 vivenciada em todo o Brasil. Em seguida, as temáticas propostas para os vídeos foram divididas por duplas que deveriam divulgá-los semanalmente nas redes sociais do projeto e aos profissionais das unidades básicas de saúde (UBS) do município de Três Lagoas.

As temáticas abordadas eram escolhidas em comum acordo entre as coordenações do Núcleo de Telessaúde da Secretaria do Estado de Saúde de Mato Grosso do Sul (SES/ MS), da Secretaria Municipal de Saúde (SMS) de Três Lagoas e da unidade de telessaúde do CPTL/UFMS. Foram contemplados temas como: saúde mental durante o isolamento social, formas de transmissão do coronavírus, violência doméstica na pandemia, cuidados clínicos destinados a grupos de risco, uso de equipamentos de proteção individual (EPI), isolamento domiciliar, entre outros.
A montagem e edição dos vídeos ficaram a cargo da unidade de telessaúde do CPTL/UFMS seguindo os roteiros previamente submetidos à aprovação da equipe da SMS de Três Lagoas e do Telessaúde da SES/MS, antes da edição final do material. Não obstante, o Núcleo de Telessaúde era responsável por promover a divulgação por meio de seu canal oficial, o que foi corroborado pelo compartilhamento nas redes sociais de nossa unidade.

Durante o processo de produção dos vídeos curtos, pelo fato de o projeto não contar com financiamento voltado à contratação de ferramentas digitais mais robustas, a unidade lançou mão de ferramentas gratuitas e acessíveis, sem abrir mão da qualidade do material. A falta de um ambiente adequado de gravação contribuiu para a falta de padronização dos ambientes de gravação, dos estilos e da qualidade do som e imagem. Entretanto, mesmo executados por ferramentas não padronizadas, consideramos exitosa a missão de ofertar um conteúdo de qualidade a toda a comunidade, com informações de caráter científico, mas com linguagem acessível à populaçãoalvo, um dos intuitos do nosso projeto.

Assim, a produção de vídeos curtos no projeto nos possibilitou maior conhecimento sobre os tipos e as especificidades de ferramentas digitais disponíveis, bem como sobre o manuseio delas. Nesse sentido, podemos destacar que a experiência foi de grande relevância para a nossa formação, dada a necessidade de profissionais com habilidades para a comunicação em saúde baseada em evidência nas redes sociais, a fim de promover uma orientação adequada em um momento atípico e de incertezas.

No tocante às teleconsultorias, foram realizadas de maneira assíncrona, devido às dificuldades de sincronização entre os horários do solicitante e dos teleconsultores docentes. Elas eram iniciadas por profissionais da APS e encaminhadas aos teleconsultores docentes credenciados ao Núcleo de Telessaúde, que discutiam as questões apresentadas em formato de estudos clínicos conosco, e, por fim, o retorno das questões ocorria em até 72 horas.

Lidar com casos reais, de modo a auxiliar os profissionais requerentes nas etapas de diagnóstico, tratamento/terapêutica, uso e prescrição de medicamentos, entre outras ações assistenciais, possibilitou-nos aguçar as atividades de pesquisa e o manejo da informação de modo democrático, e integrar o conhecimento teórico aprendido na graduação a resoluções práticas no auxílio aos diferentes profissionais da equipe de saúde - agentes comunitários de saúde (ACS), enfermeiros, médicos, nutricionistas, psicólogos, entre outros.

Nossa participação na construção da resposta às teleconsultorias nos permitiu o aprimoramento de habilidades para o raciocínio clínico e o conhecimento dos principais 
motivos de encaminhamentos de usuários dos serviços da APS para serviços especializados. Percebemos também que a maioria das dúvidas dos profissionais da APS poderia ser manejada pela equipe da unidade, com auxílio da telessaúde, sem a necessidade de encaminhamentos à atenção especializada, tornando o atendimento da APS mais resolutivo e diminuindo as filas de espera.

As "perguntas da semana" eram encaminhadas pelo Núcleo de Telessaúde a partir do levantamento dos questionamentos mais frequentes das teleconsultorias. Utilizamos como estratégia de divulgação em nossa unidade de telessaúde do CPTL/UFMS, além das postagens oficiais no site do Telessaúde MS na aba "Perguntas da Semana", a disponibilização de um chat de esclarecimentos de dúvidas nas redes sociais, principalmente Instagram e Facebook - com posts, stories e quiz acerca do assunto tratado. A partir dessa ferramenta, outros estudantes de Medicina e pessoas da comunidade poderiam direcionar suas dúvidas à equipe da unidade de telessaúde do CPTL/UFMS.

Também participamos da elaboração de uma SOF publicada no site da BVS da Organização Pan-Americana da Saúde (Opas), na área de ginecologia e obstetrícia. Trata-se de uma ferramenta necessária para a divulgação na comunidade científica de temas de profunda relevância que podem vir a solucionar consultas médicas futuramente, na forma presencial ou não.

Por fim, constatamos que o uso das redes sociais para o compartilhamento de informações e interação possibilitounos experienciar e perceber a importância desse espaço de comunicação e aprendizagem para a nossa formação. Entretanto, como ponto negativo, percebemos uma baixa adesão da população a esse recurso, talvez pela problemática do acesso à internet. Apesar de termos alcançado a marca de 413 seguidores no Instagram um mês após a criação da página e, em março de 2021, contarmos com 431 seguidores, ainda sentimos que havia certa restrição no engajamento das pessoas com as nossas publicações. No Facebook, a página "Telessaúde UFMS Três Lagoas" angariou 524 curtidas, ou seja, 524 pessoas acompanhavam diretamente as publicações desempenhadas nessa rede social, entretanto qualquer pessoa que tivesse uma conta conseguiria visualizar o conteúdo por nós publicado.

\section{DISCUSSÃO}

A experiência vivenciada possibilitou o aprimoramento de conhecimentos teórico-práticos acerca de ferramentas para o desenvolvimento de materiais educativos digitais. Tal conhecimento torna-se de suma relevância dado o avanço das TIC, as quais são um importante meio de acesso às informações em saúde. A valorização das TIC na área de educação em saúde permite o desenvolvimento de iniciativas pedagógicas de saúde criativas, inovadoras e ousadas, as quais fortalecem a interface entre comunicação, ciência e sociedade ${ }^{13}$.

Não obstante, é importante destacar a relevância de oferecimento de conteúdo com amplo respaldo científico e alto nível de evidências científicas. Para isso, foram consideradas as recomendações descritas no Protocolo de Respostas a Teleconsultorias do Ministério da Saúde ${ }^{10}$ e publicações em periódicos científicos disponíveis em bases de dados nacionais e internacionais. Esse fato mostrou-se imprescindivelmente necessário, sobretudo durante o cenário epidêmico, uma vez que a Covid-19 é uma doença sem precedentes cuja maioria da população ainda não compreende sua causa e efeito, cenário perfeito para a circulação de falsas informações, que muitas vezes não são verificadas e geram dúvidas em seus interlocutores. Assim, com o intuito de combater a prática de fake news, torna-se relevante a criação de espaços de comunicação e esclarecimento de dúvidas nos núcleos de telessaúde com perguntas e respostas, de modo a evitar a disseminação de notícias falsas sobre a Covid-19 ${ }^{14}$.

O processo de construção dos vídeos e materiais educativos possibilitou o aprofundamento teórico sobre as temáticas a partir da pesquisa em bases de dados científicas, o que resultou na capacitação dos envolvidos para o reconhecimento das bases e das melhores evidências científicas. O conhecimento sobre a medicina baseada em evidências (MBE) e sobre a pesquisa em bases de dados é de suma relevância para o protagonismo do estudante no processo de aprendizagem, trazendo implicações para a excelência dos futuros profissionais. Um estudo realizado com estudantes de Medicina de uma universidade no estado de Pernambuco constatou que mais de $50 \%(68,3 \%)$ ainda se identificam com a pesquisa em livros físicos e que a maioria não tem recebido treinamento em MBE ou em técnicas de pesquisa bibliográfica em bases especializadas ${ }^{15}$.

Além disso, objetivou-se efetivar a união entre a comunidade científica e a leiga, de modo a reforçar os laços entre sociedade e universidade, e garantir um retorno social das atividades desempenhadas pela universidade pública. Nesse contexto, propôs-se que o conteúdo - seja ele de caráter técnico ou cotidiano - deveria ser abordado de maneira simplificada por meio de uma linguagem simples, sem rebuscamento e de fácil compreensão ${ }^{6}$.

O compartilhamento das informações pelas redes sociais teve como alvo alcançar o maior engajamento possível e respeitar as medidas de distanciamento do cenário epidemiológico. Sobre isso, um estudo realizado com 310 estudantes de Medicina, com a finalidade de examinar as contribuições das redes sociais (principal plataforma de 
veiculação dos trabalhos do Núcleo) no ensino na graduação em Medicina, identificou como vantagens o compartilhamento de informações, a interação entre pessoas, a facilidade e a rapidez, e a formação de grupos ${ }^{16}$.

O desenvolvimento de experiências no projeto permitiu sobretudo a discussão de conteúdos previstos no currículo do curso de Medicina em formato de teleconsultorias, de modo a favorecer a formação do acadêmico. Portanto, deve-se ressaltar a necessidade de inclusão do ensino da telemedicina nos currículos dos cursos de graduação ${ }^{11}$.

Além de evitar que muitos pacientes se exponham a possível contaminação pelo vírus ao buscarem um serviço de saúde em um cenário de ampla propagação de moléstias infectocontagiosas, é válido reforçar o papel social das teleconsultorias, que levam atendimento a pacientes que vivem longe de locais de saúde, permitindo que evitem longas viagens e custos mais elevados ${ }^{17}$.

Não obstante, é extremamente válido destacar a importância do preenchimento do requisito de educação permanente nas teleconsultorias. Por meio dele, cumpriu-se o objetivo de fomentar a educação em saúde, de forma a guiar o profissional requerente a realizar pesquisas futuras nos bancos de dados, promovendo a educação médica continuada2,18. Outrossim, constatou-se que tal fato teve repercussão direta com o decorrer do projeto, com reflexo nas condutas que deveriam ser tomadas pelo profissional antes de solicitar o preenchimento. Além disso, fornecemos com frequência orientações sobre a requisição e o preenchimento correto das teleconsultorias, sobretudo quando havia dados insuficientes ou no caso de algumas informações relevantes que poderiam ser mencionadas. Todo esse processo tinha como propósito promover um tratamento holístico destinado ao paciente e a prática da medicina preventiva.

A produção das SOFs (disponíveis no site do Telessaúde MS, com acesso amplo e gratuito) acaba por fortalecer os vínculos com a APS e a população em geral, pelo fato de se basearem nas melhores evidências científicas e clínicas, contribuírem para o papel ordenador desse nível de atenção e sanarem dúvidas frequentes que são interpeladas aos profissionais de saúde que participaram direta ou indiretamente do projeto ${ }^{19}$.

Por fim, cabe mencionar a falta de engajamento da população identificada na experiência aqui apresentada, o que também se verificou em outro estudo brasileiro ${ }^{20}$, já que a abordagem realizada por acadêmicos e não profissionais formados foi apontada como uma barreira para uma maior adesão.

Apesar de o uso da telessaúde ainda ser baixo no Brasil, houve um grande crescimento desse tipo de serviço no período de pandemia da Covid-19, mediante a aprovação dessa ferramenta e o estímulo à utilização dela, a qual ainda não se encontrava regulamentada no país. Acredita-se que o uso bemsucedido da telessaúde neste período poderá contribuir para uma maior aceitação pública e governamental no país e para maiores investimentos na formação de profissionais para o uso de tais tecnologias ${ }^{14}$.

\section{CONSIDERAÇÕES FINAIS}

Com base no que foi exposto neste artigo, conclui-se que a participação de estudantes de Medicina nas atividades de telessaúde resultou em um saldo muito positivo na formação profissional e pessoal dos envolvidos. O envolvimento de discentes em ações voltadas para a minimização da exposição da população ao vírus e para a maior efetividade dos serviços da APS durante a pandemia contribuiu para reduzir a angústia dos estudantes que se encontram impossibilitados de realizar aulas presenciais neste período.

Um dos intuitos da telessaúde foi estabelecer vivências entre acadêmicos e profissionais de saúde na execução de habilidades que devem ser desempenhadas e que são requisitos para uma boa formação médica, além de permitir a formação de um canal de comunicação confiável e científica com a comunidade em geral. Com isso, estabeleceu-se um meio de transmitir informações para a prática da MBE, além de aguçar o senso crítico tanto dos alunos quanto dos requerentes das teleconsultorias.

Ademais, fomentou-se o aprimoramento dos conhecimentos dos estudantes sobre as TIC que, com certeza, farão parte do futuro cotidiano profissional. Considerando isso, torna-se necessária a inclusão de conteúdos teórico-práticos em telessaúde nas grades curriculares dos cursos de Medicina, a fim de fornecer aos acadêmicos conhecimento acerca dessa modalidade de serviço e promover a vivência de experiências de formação como as aqui relatadas.

\section{CONTRIBUIÇÃO DOS AUTORES}

Matheus Augusto Obici Massucato participou da produção e edição textual, e da revisão final do manuscrito. Ana Luísa Green Ribeiro participou da produção e edição textual. Juliana Dias Reis Pessalacia e Vinicius de Jesus Rodrigues Neves participaram da edição e revisão textual, e orientaram a elaboração do estudo. Vânia Paula Stolte-Rodrigues participou da edição e revisão textual.

\section{CONFLITO DE INTERESSES}

Declaramos não haver conflito de interesses.

\section{FINANCIAMENTO}

Declaramos não haver financiamento. 


\section{REFERÊNCIAS}

1. Shirke MM, Shaikh SA, Harky A. Implications of telemedicine in oncology during the Covid-19 pandemic. Acta Biomed. 2020 Sept 7;91(3):e2020022. doi: 10.23750/abm.v91i3.9849.

2. Pollock K, Setzen M, Svider PF. Embracing telemedicine into your otolaryngology practice amid the Covid-19 crisis: an invited commentary. Am J Otolaryngol. 2020;41(3):e102490. doi: 10.1016/j.amjoto.2020.102629.

3. Lancu AM, Kemp MT, Alam HB. Unmuting medical students' education: utilizing telemedicine during the Covid-19 pandemic and beyond. J Med Internet Res. 2020 July 20;22(7):e19667. doi: 10.2196/19667.

4. Hilburg R, Patel N, Ambruso S, Biewald MA, Farouk SS. Medical education during the coronavirus disease-2019 pandemic: learning from a distance. Adv Chronic Kidney Dis. 2020;27(5):412-7. doi: 10.1053/j.ackd.2020.05.017.

5. Pitt MB, LiST, Klein M. Novel educational responses to Covid-19: what is here to stay? Acad Pediatr. 2020;20(6):733-4. doi: 10.1016/j.acap.2020.06.002.

6. Brasil. Portaria $n^{\circ} 467$, de 20 março de 20. Dispõe, em caráter excepcional e temporário, sobre as ações de Telemedicina, com o objetivo de regulamentar e operacionalizar as medidas de enfrentamento da emergência de saúde pública de importância internacional previstas no art. $3^{\circ}$ da Lei n० 13.979 , de 6 de fevereiro de 2020, decorrente da epidemia de Covid-19 [acesso em 14 abr 2021]. Disponível em: https://bvsms.saude. gov.br/bvs/saudelegis/gm/2020/prt0467_23_03_2020_extra.html.

7. Rienits $\mathrm{H}$, Teuss $\mathrm{G}$, Bonney A. Teaching telehealth consultation skills. Clinic Teach 2015;13(2):119-23. doi: 10.1111/tct.12378.

8. Yaghobian S, Ohannessian R, lampetro T, Riom I, Salles N, de Bustos EM, et al. Knowledge, attitudes and practices of telemedicine education and training of French medical students and residents. J Telemed Telecare. 2020 June 9;0(0):1-9. doi: 10.1177/1357633X20926829.

9. Damasceno RF, Caldeira AP. Fatores associados à não utilização da teleconsultoria por médicos da Estratégia Saúde da Família. Ciênc. Saúde Colet. 2019;24(8):3089-98. doi: 10.1590/1413-81232018248.28752017.

10. Ministério da Saúde, Universidade Federal do Rio Grande do Sul. Manual de telessaúde para a atenção básica/atenção primária à saúde: protocolo de solicitação de teleconsultorias. Porto Alegre: Universidade Federal do Rio Grande do Sul; Brasília: Ministério da Saúde; 2013.
11. Rallis KS, Allen-Tejerina AM. Tele-oncology in the Covid-19 era: are medical students left behind? Trends Cancer. 2020 Oct;6(10):811-2. doi: 10.1016/j. trecan.2020.08.001.

12. Kopp AR, Rikin S, Cassese T, Berger MA, Raff AC, Gendlina I. Medical student remote eConsult participation during the Covid-19 pandemic. BMC Med Educ. 2021 Feb 22;21:e120. doi: 10.1186/s12909-021-02562-6.

13. França $T$, Rabello ET, Magnago C. As mídias e as plataformas digitais no campo da educação permanente em saúde: debates e propostas. Saúde Debate. 2019;43(esp 1):106-15. doi: 1590/0103-11042019s109.

14. Caetano R, Silva AB, Guedes AC, Paiva CC, Ribeiro GR, Santos DL, et al. Desafios e oportunidades para telessaúde em tempos da pandemia pela Covid-19: uma reflexão sobre os espaços e iniciativas no contexto brasileiro. Cad Saude Publica. 2020;36(5):e00088920. doi: 10.1590/0102$311 \times 00088920$.

15. Lorena SB, Andrade MM, Arcoverde AH, Vilela LS, Mota LA, Lorena Sobrinho JE. Análise do acesso à informação acadêmica entre estudantes de Medicina inseridos numa metodologia ativa de aprendizagem. Rev Bras Educ Med. 2019;43(4):176-86. doi: 10.1590/1981-52712015v43n4rb20190037.

16. Purim KM, Tizzot EA. Protagonismo dos estudantes de Medicina no uso do Facebook na graduação. Rev Bras Educ Med. 2019;43(1):187-96. doi: 10.1590/1981-52712015v43n1rb20180139.

17. Lee MS, Nambudiri V. Integrating telemedicine into training: adding value to graduate medical education through electronic consultations. J Grad Med Educ. 2019;11(3):251-4. doi: 10.4300/JGME-D-18-00754.1.

18. Maeyama MA, Calvo MC. A integração do telessaúde nas centrais de regulação: a teleconsultoria como mediadora entre a atenção básica e a atenção especializada. Rev Bras Educ Med. 2018;42(2):63-72. doi: 10.1590/1981-52712015v42n2rb20170125.

19. Guimarães EM, Godoy SC, Silva LF, Trindade, RM. A Segunda Opinião Formativa como instrumento de educação permanente na telenfermagem. J Bras Telessaúde. 2018;5(1):247-8.

20. Dimer NA, Canto-Soares ND, Santos-Teixeira LD, Goulart BNG. The Covid-19 pandemic and the implementation of telehealth in speech-language and hearing therapy for patients at home: an experience report. Codas. 2020 June 22;32(3):e20200144. doi: 10.1590/2317-1782/20192020144. 